\title{
TETRALOGY OF FALLOT IN A FRIESIAN HEIFER
}

\author{
BY \\ EDWARD W. FISHER AND HUGH M. PIRIE \\ From the University of Glasgow Veterinary Hospital, Glasgow \\ Received May 1, 1963
}

Only three cases of tetralogy of Fallot in cattle have been described (Hahn, 1908; Jasper, 1948; Cordy and Ribelin, 1950). None of these reports has included detailed descriptions of the clinical and physiological disturbances associated with this anomaly. The following case is reported since for the first time in cattle it was possible, using specialized techniques, to establish the diagnosis in life with reasonable certainty.

\section{Methods of Examination}

In addition to clinical and pathological examinations the following examinations were carried out.

Electrocardiograms. Electrocardiograms were taken using the standard limb leads of Einthoven and unipolar limb leads with the right arm electrode attached to the right foreleg, the left arm electrode to the left foreleg, the left leg electrode attached to the left hind leg, and the right leg electrode attached to the right hind leg. The cardiograph used was a Siemens Cardirex 6.*

Heart Sound Recordings. Using the Cardirex 6, graphic records were taken of the heart sounds at five different frequency bands, together with a lead II electrocardiogram for timing.

Dye Dilution Curves. By means of a long polythene catheter inserted into the jugular vein it was possible to inject volumes of Evans Blue dye into the right atrium, right ventricle, and pulmonary artery. The appearance of the dye in the arterial circulation was followed by removing arterial samples at one second intervals and measuring the concentration of dye in these samples.

Blood Pressures. Pressure recordings were made from the right side of the heart by inserting a polythene catheter down the jugular vein. The catheter was connected to a sensitive inductance manometer, $\dagger$ the output of which was fed into the Cardirex 6 for graphic recording.

\section{CASE REPORT}

The subject, No. 19429, was admitted to the Veterinary Hospital at the age of 9 months with a history of not thriving. It was dull in demeanour, in poor condition, weighed $148 \mathrm{~kg}$., and had large subcutaneous abcesses behind the left shoulder and upon the left patella. The feet were very overgrown. At rest the respiratory rate was 50 a minute and the heart rate was 100 a minute. On auscultation, bronchovesicular respiratory sounds were heard. Palpation of the chest revealed a præcordial thrill on the left-hand side and a distinct apex beat on the right-hand side. On percussion there was an increased area of cardiac dullness on both sides of the chest.

The first heart sound was obscured by a gross systolic murmur which was most intense at the pulmonic area, that is under the elbow at the third intercostal space, about $5 \mathrm{~cm}$. above the level of the olecranon. The pulse volume was very poor. Cyanosis was detectable at rest on the mucous membrane of the eye, the mouth, and the vulva. When the animal was exercised by being run for a distance of 150 yards it became much more cyanotic and dyspnœa developed.

Hæmatological examination showed polycythæmia with a hæmoglobin concentration of $20.6 \mathrm{~g}$. (normal 9-11 g.) and a red blood cell count of 14.3 million (normal 5-8 million). The white blood cell count and the plasma protein were normal.

\footnotetext{
* Supplied by Sierex Limited, London.

$\dagger$ Elema Schonander Limited, Stockholm, Sweden.

H
} 


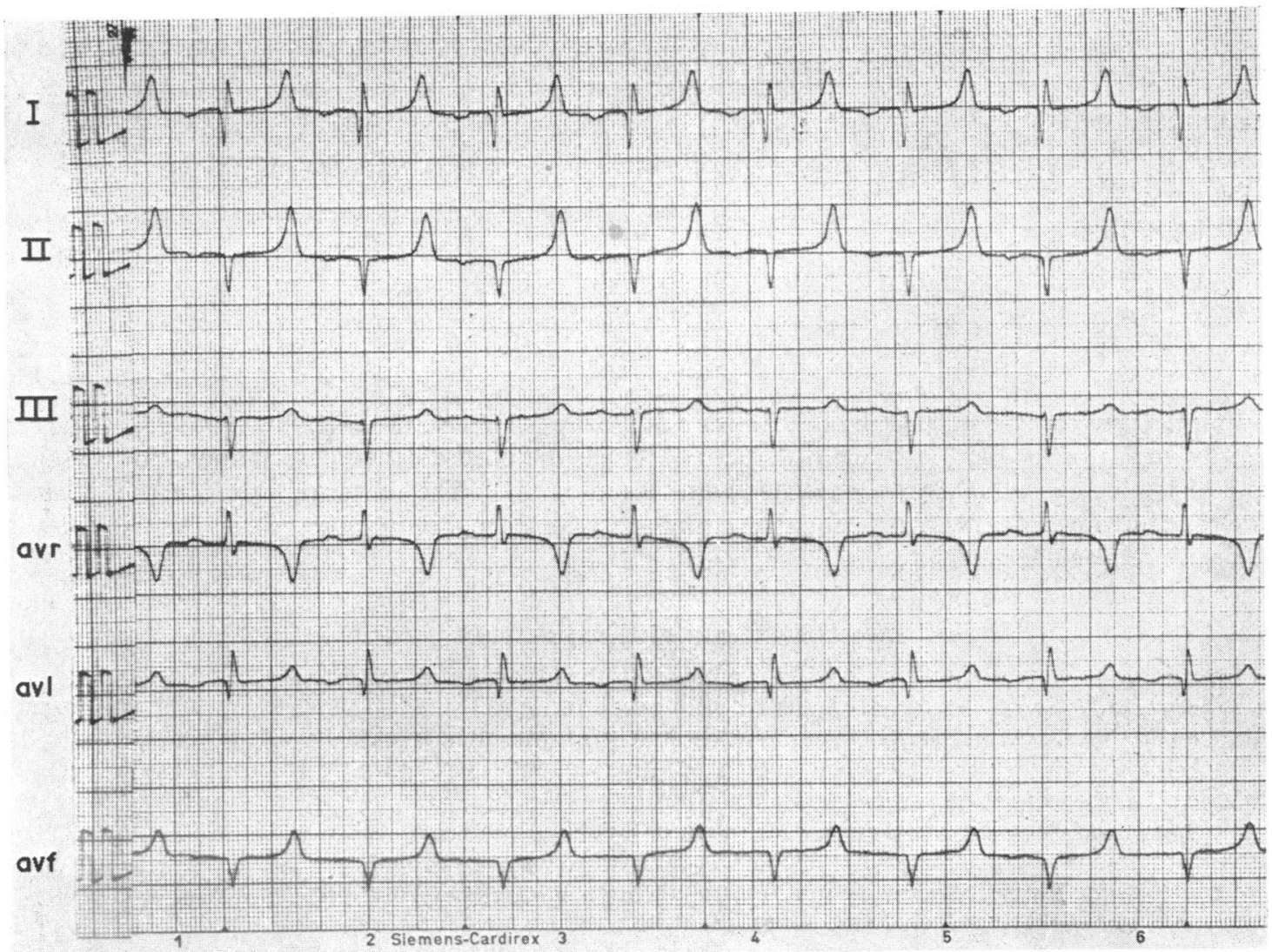

FiG. 1.-Electrocardiogram of 19429 (speed $50 \mathrm{~mm}$./sec.). aVR=unipolar limb lead, right fore; aVL =unipolar limb lead, left fore; $\mathrm{aVF}=$ unipolar limb lead, left hind.

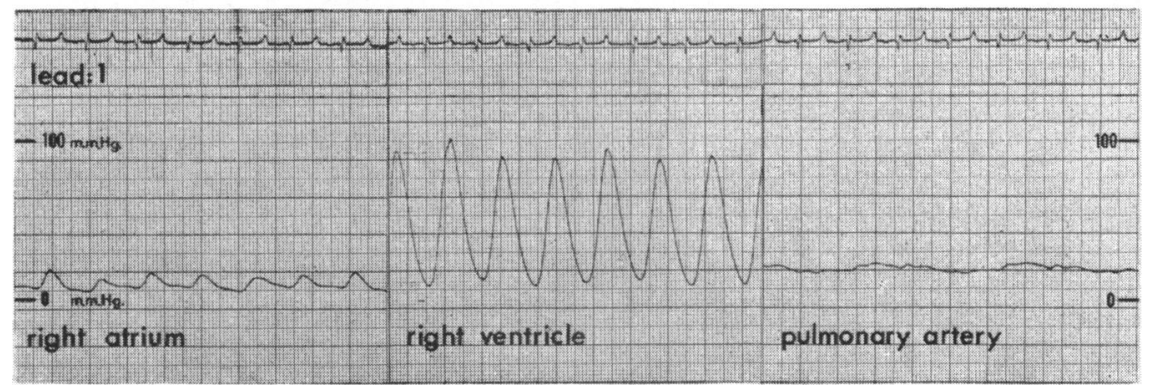

Fig. 2.-Pressure curves right heart, 19429.

The animal was kept for 10 months. During this time it showed no change in its cardiac conditon. Body weight increased from 148 to $287 \mathrm{~kg}$. when it was slaughtered.

Electrocardiograms. The cardiogram (Fig. 1) showed large complexes when compared with normal cattle, suggesting cardiac hypertrophy, while the direction of the QRS complexes in limb leads and unipolar leads suggested that this hypertrophy was on the right side of the heart. Negative $\mathbf{P}$ waves present in leads I, II, and aVL are not usually seen in cattle, but their significance is unknown.

Pressure Recordings. The pressure recordings of the right side of the heart (Fig. 2) showed a rise in 
TABLE I

Catheterization Data (Mm. Hg)

\begin{tabular}{l|r|c|c|c}
\hline & \multicolumn{2}{|c|}{ Normal heifer } & \multicolumn{2}{c}{ Fallot's tetralogy } \\
\cline { 2 - 5 } & Systolic & Diastolic & Systolic & Diastolic \\
\hline Right atrium & 5 & 0 & 19 & 5 \\
Right ventricle & 53 & 0 & 93 & 12 \\
Pulmonary artery & 45 & 19 & 23 & 16 \\
Carotid artery & 180 & 130 & 105 & 88 \\
\hline
\end{tabular}

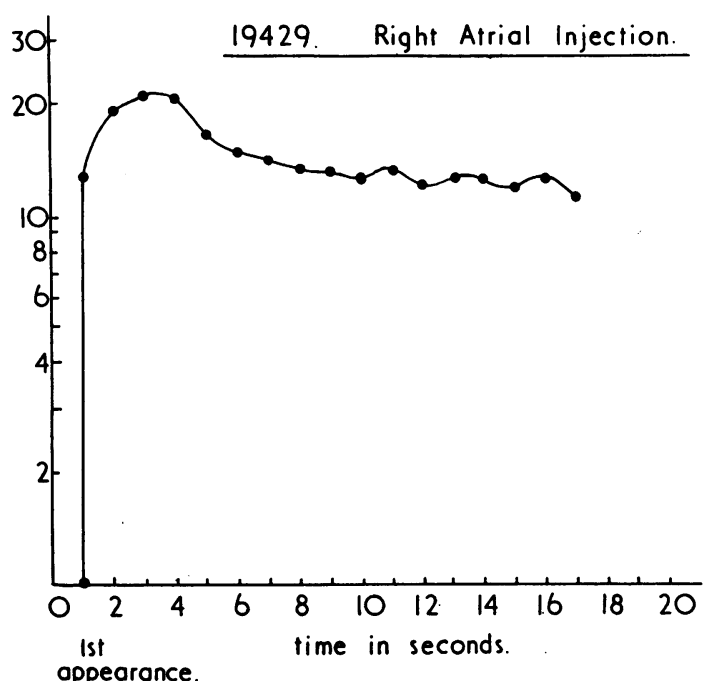

A

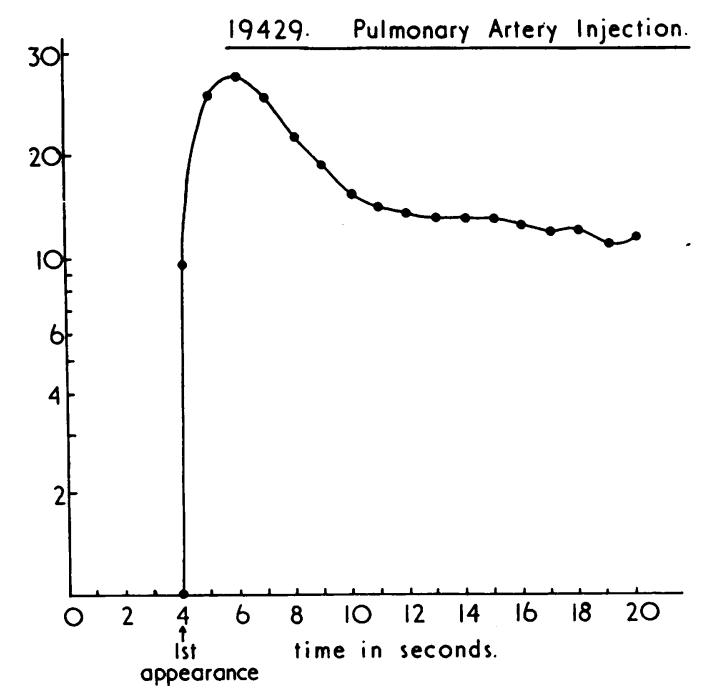

C

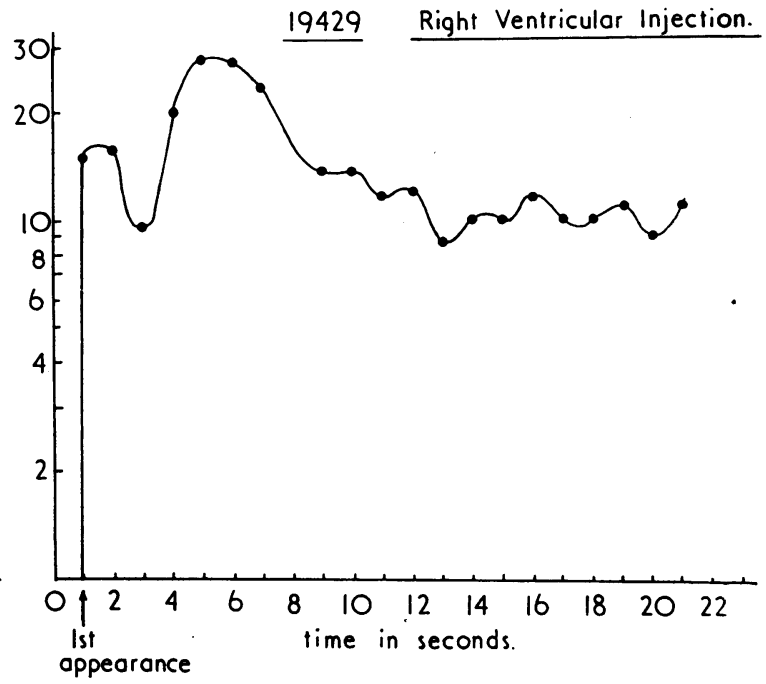

B

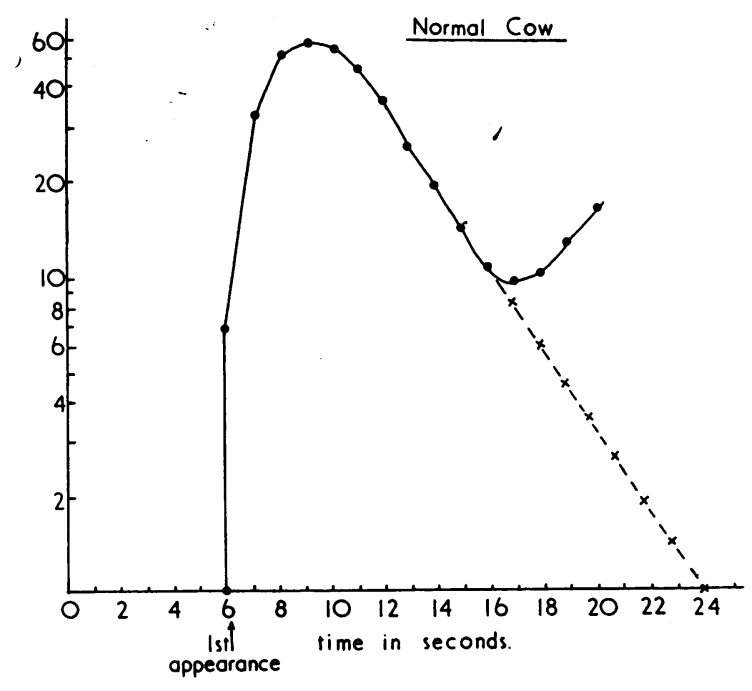

D

FIG. 3.-(A) Dye dilution curve, 19429. Right atrial injection. (B) Dye dilution curve, 19429. Right ventricular injection. (C) Dye dilution curve, 19429. Pulmonary artery injection. (D) Dye dilution curve, normal cow. 
right atrial pressure, an increased right ventricular pressure, and a lowered pulmonary arterial pressure. The values found are given in Table $\mathrm{I}$, where they are compared with a normal series of pressures found by Doyle et al. (1960). It will be observed that the arterial pressure was also below normal and that there was a low pulse pressure.

In cattle of this size it is not possible to localize the catheter tip by fluoroscopy. The position of catheters is known from experiments whereby catheters were fixed at sites from which particular pulse curves were obtained. These catheter positions were then determined at autopsy. On the basis of this previous experience the pressures obtained from this animal were known to be valid for the sites stated.

In addition, dye dilution curves obtained by the injection of Evans Blue at these sites and the first appearance times of the dye in the brachial artery gave further evidence of the positions of the catheter tips in this animal.

Dye Dilution Recordings. The dye dilution curves, which were obtained by plotting the varying concentrations of Evans Blue in plasma against the time of their appearances in the brachial artery, are shown in Fig. 3A, B, and C. A curve from a normal cow is also illustrated (Fig. 3D). The right atrial and right ventricular injections of dye produced curves indicating abnormal mixing of blood within the heart. Moreover, the time of first appearance of the dye was in each case more rapid than normal, suggesting a right-toleft shunt. The injection of dye into the pulmonary artery produced a dye dilution curve which was normal in shape except that the first recirculation was not so obvious as in a normal animal. The time of first appearance of dye from injection into the pulmonary artery was also normal. The delayed downstroke of the dye dilution curves $3 \mathrm{~A}, \mathrm{~B}$, and $\mathrm{C}$ suggested that in addition a left-to-right shunt existed. Therefore from these curves it was concluded that there was a gross abnormality of blood flow through the heart, which included a right-to-left shunt at the level of the ventricles and a left-to-right shunt at a higher level.

Heart Sound Recordings. These recordings, which are illustrated in Fig. 4, demonstrate the systolic murmur; and this was demonstrable at all frequencies. The pulmonary component of the second heart sound was not recorded. This abnormality is noted in Fallot's tetralogy in man (Wood, 1956).

Diagnosis. In arriving at a diagnosis, the following facts were considered significant. In this young animal there was clinical evidence of cardiac hypertrophy, a loud systolic murmur most intense at the pulmonary area, in combination with tachypnœa and cyanosis at rest. These findings, together with electrocardiographic evidence of right ventricular hypertrophy, indicated the presence of a major congenital cardiac anomaly. The dye dilution curves demonstrated a defect in the ventricular septum with a right-to-left shunt, while the pressure recordings from the right side of the heart showed the presence of pulmonary stenosis. Thus cyanosis, right ventricular hypertrophy, a right-to-left shunt in the ventricles, and pulmonary stenosis led to diagnosis of the tetralogy of Fallot.

Autopsy. The heart was enlarged, weighed $1620 \mathrm{~g}$., and when viewed anteriorly the apex was rounder than normal. The gross disproportion in size between the hypoplastic pulmonary trunk and the dilated ascending aorta was obvious (Fig. 5). The wall of the pulmonary trunk was less than half the thickness of the wall of the aorta. The ductus arteriosus, which was patent, also had a thin wall and connected with the beginning of the left pulmonary artery (Fig. 5). The dilated right atrium was slightly hypertrophied, and the foramen ovale, although functionally competent, was anatomically patent.

The wall of the right ventricle, which was grossly hypertrophied (Fig. 6), was as thick as the left ventricle, and the lumen of the ventricle extended ventrally to the apex farther than normal. High in the ventricular septum was a large elliptical ventricular septal defect $3.5 \times 1.5 \mathrm{~cm}$. with its long axis horizontally situated (Fig. 6). Above the ventricular septal defect the aorta was dextroposed so that its anterior cusp was on the right side of the ventricular septum when viewed from above. In the left ventricle the ventricular septal defect appeared below the anterior cusp of the aorta. The aortic arch was on the left side.

The aortic valve was dilated and had three cusps which were larger than normal. The bones of the heart were well developed and the coronary arteries were normal. The aorta itself was dilated to the origin of the ductus arteriosus and the proximal part of the brachiocephalic trunk was also dilated.

The pulmonary valve was less than half the diameter of the aortic valve and had only two large cusps (Fig. 7) which were excessively large for the size of the valve and were anterior and posterior in position. Below the pulmonary valve there was an infundibular type of stenosis which had produced a narrow curving channel $5 \mathrm{~cm}$. long, forming the outlet from the right ventricle to the pulmonary trunk (Fig. 6). There was no ring of fibrous tissue at the proximal opening of the channel which had thick muscular walls and was dilated immediately below the pulmonary valve, forming an infundibular chamber. 


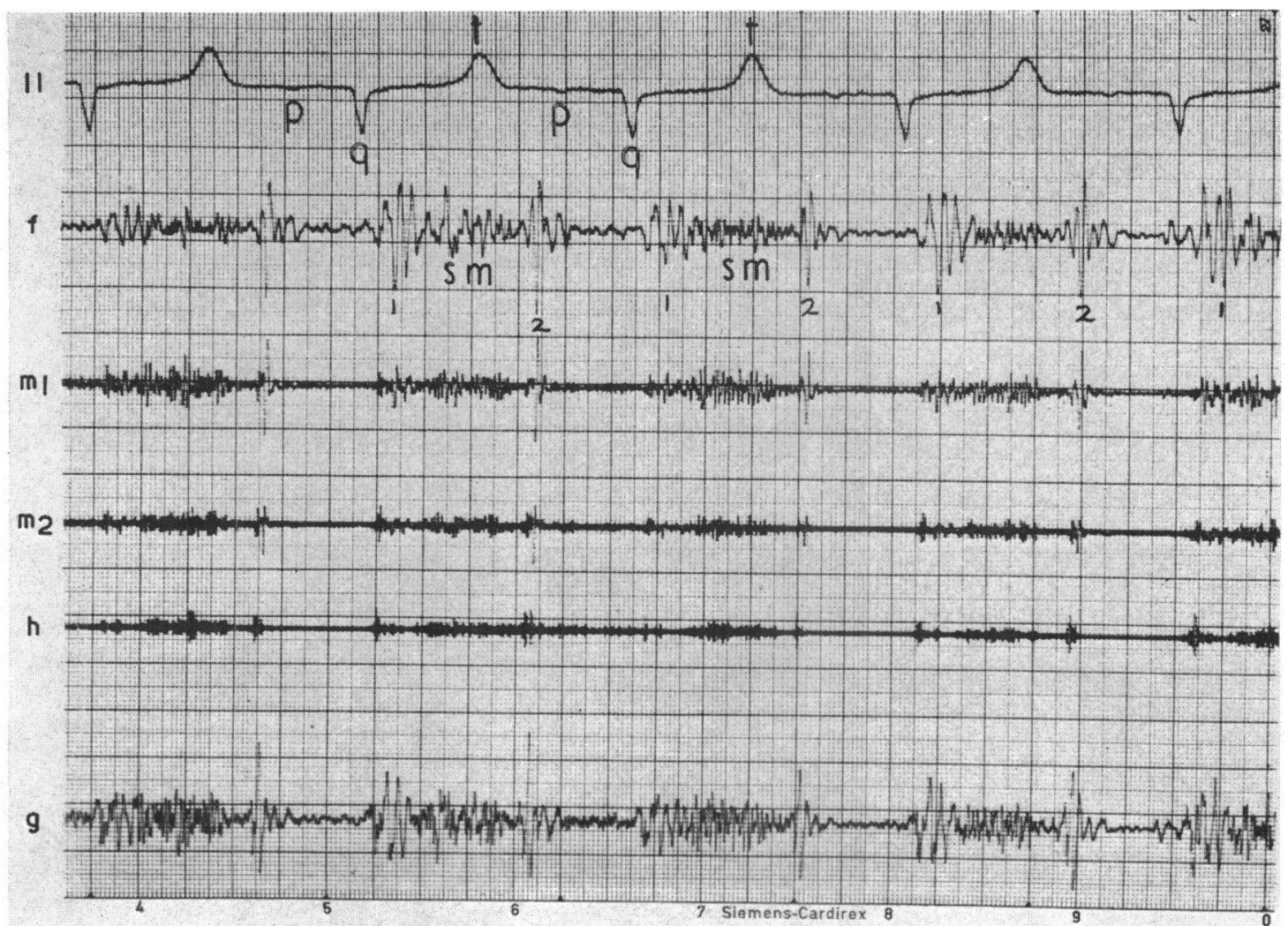

FIG. 4.-Heart sound records, 19429. Top row, electrocardiogram, lead II; second row, f, low frequency heart sound; $\mathrm{m} 1$, medium frequency first heart sound; $\mathrm{m} 2$, medium frequency second heart; $h$, high frequency sound; g, stethoscopic heart sound; and sm, systolic murmur.

The left atrium was normal and the wall of the left ventricle was not hypertrophied.

A large abscess $22 \times 22 \times 15 \mathrm{~cm}$. involving the diaphragmatic surface of the liver in its dorsal half and the diaphragm itself was found. The diaphragmatic lobes of the lungs were adherent to the thoracic surface of the diaphragm over the abscess. Scattered throughout the liver were several smaller abscesses $2 \mathrm{~cm}$. in diameter, either showing through the liver capsule as white circular convex areas or hidden in the liver parenchyma. No abscesses were found in the brain. The abscesses behind the left shoulder and upon the left patella had healed by the time the animal came to autopsy, and only increased amounts of fibrous connective tissue at these sites indicated where the lesions had been.

A few small pale wedge-shaped areas were seen in the cortices of the kidneys, along with some moderately large hæmorrhagic infarcts. The bone marrow in all of the vertebræ and sternebrae bodies was bright red and soft.

Histology. Histological examination of the heart showed that although there was considerable variation in the width of the fibres in the right ventricle, some were wider than normal. Many fibres had large nuclei, and groups of three to four nuclei close together were frequently present. In the lungs there were several moderately heavy peribronchial accumulations of lymphocytes. Histological examination of the liver and the kidneys showed the usual changes associated with abscess formation and infarction. No significant lesions were seen in the other organs.

\section{Discussion}

Fallot's tetralogy has been described in cattle by Hahn (1908), Jasper (1948), Cordy and Ribelin (1950); in dogs by Meredith and Clarkson (1959), Glazier and Kealy (1960), Detweiler, 


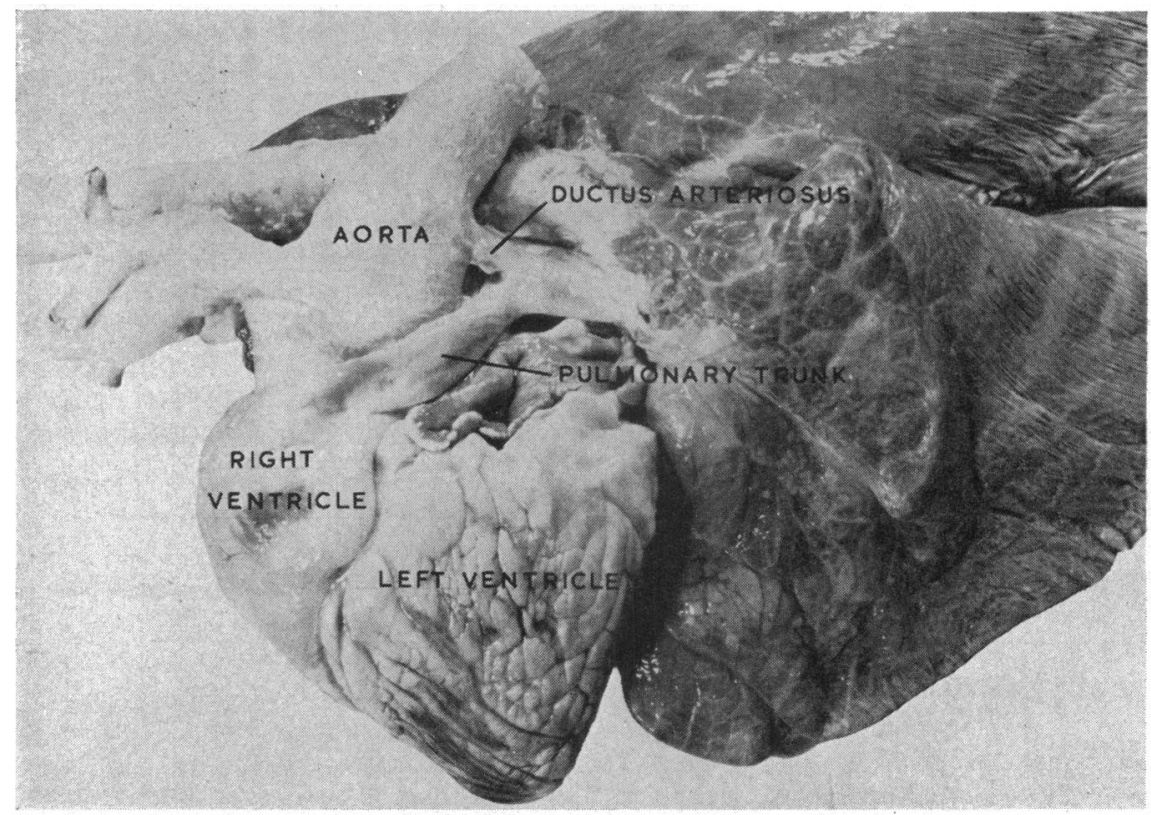

Fig. 5.-Left lateral view of heart.

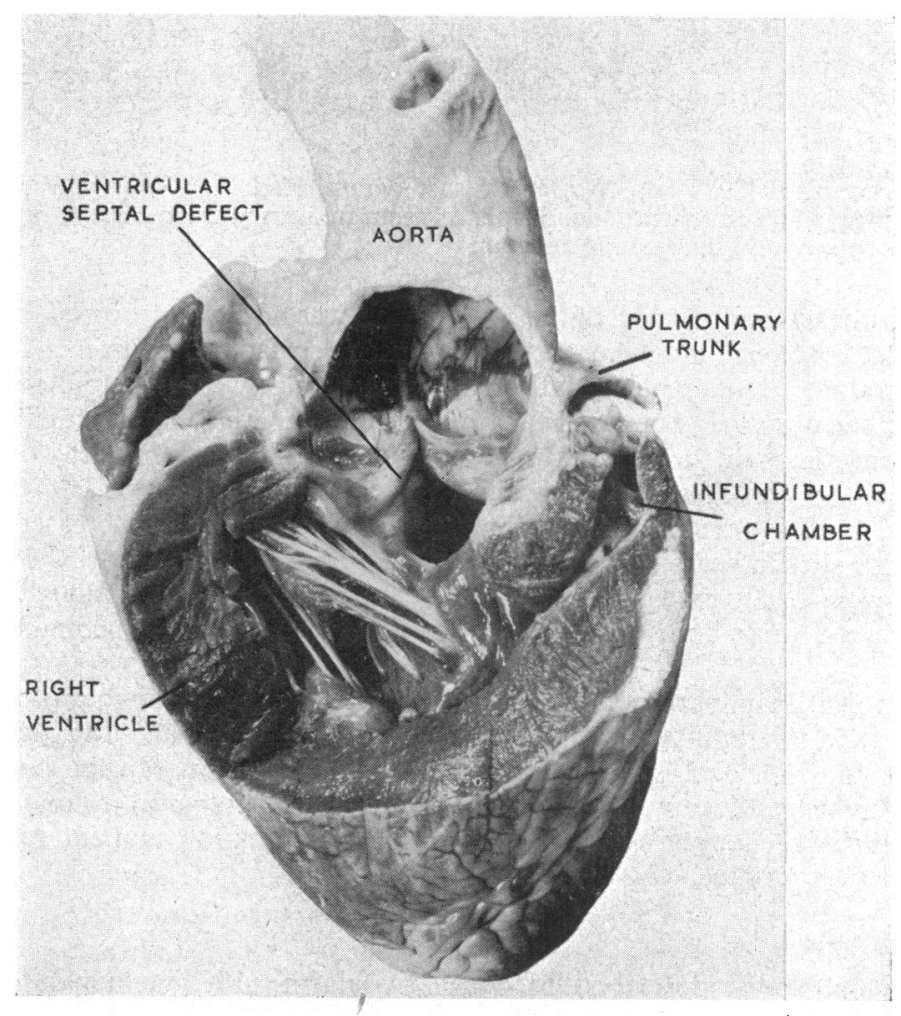

Fig. 6.-View into right ventricle. 
Hubben, and Patterson (1960); in a horse by Wensvoort (1959); and in pigs by Van Nie (1961). Only in human subjects have extensive studies been made of the clinical and functional disturbances. Cardiac catheterization has been attempted in the dog (Meredith and Clarkson, 1959), but even with fluoroscopic monitoring of the catheter tip it could not be passed through the pulmonary stenosis. Three living cases of tetralogy of Fallot in cattle have been studied by us, but only in this case was it possible to catheterize the pulmonary trunk.

In cattle the disease is similar to that described in the human patient, clinically, physiologically, and pathologically. Clinically there is cyanosis, tachycardia, a loud systolic murmur, tachypnœa with dyspnœa on exertion, and a poor volume pulse. Physiological examination of this case revealed right ventricular hypertrophy, right ventricular hypertension, pulmonary hypotension and the presence of a right-to-left shunt at the level of the ventricle. Pathologically the four features of the anomaly were found. In addition, in four

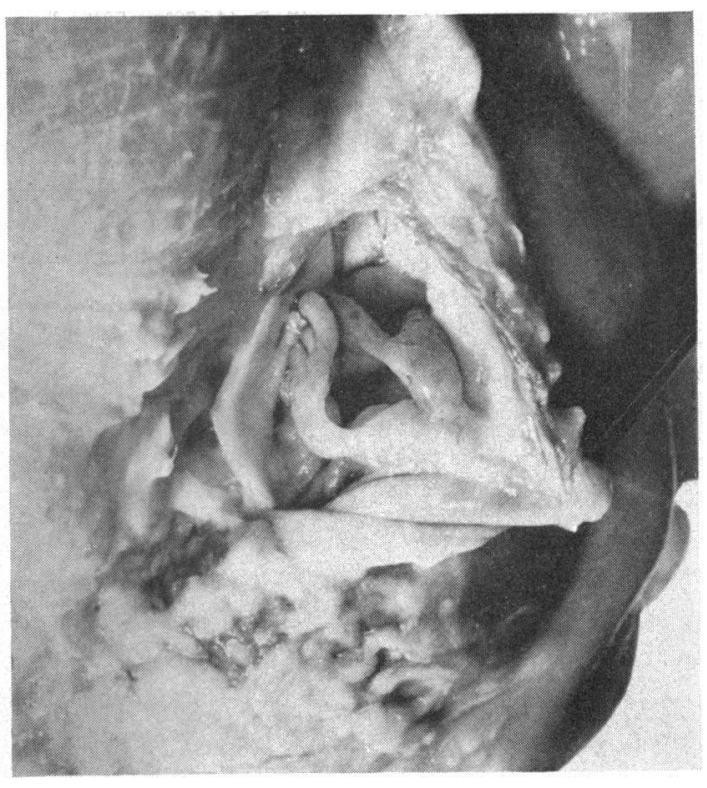

Fig. 7.-Bicuspid pulmonary valve. cases observed by us and in the case described by Jasper (1948), the ductus arteriosus was patent.

It has not been possible to find evidence of tetralogy of Fallot with persistent right aortic arch in cattle, although persistent right aortic arch as a single entity has been described (Roberts, Kennedy, and Delehanty, 1953).

It is of interest to speculate whether the peculiar overgrowing of the feet observed in this animal was analogous to the clubbing of the fingers seen in cyanotic forms of congenital heart disease in man.

Although the animal survived and gained weight in the sheltered environment of the Veterinary Hospital, the weight gain was slightly less than normal, and it is doubtful if survival would have been possible under normal farm conditions.

\section{SUMMARY}

A case of Fallot's tetralogy in a Friesian heifer is described. It was possible, for the first time in a species other than man, to arrive at the diagnosis with reasonable certainty in life, by means of cardiac catheterization, and selective injections of Evans Blue dye.

We would like to acknowledge the technical assistance of Mr. J. Murphy, M.S.I.S., and of Mrs. Helen McLeod. The photographs were taken by Mr. A. Finnie, the Veterinary School photographer, to whom we also owe thanks.

\section{REFERENCES}

Cordy, D. R., and Ribelin, W. E. (1950). Six congenital cardiac anomalies in animals. Cornell Vet., 40, 249.

Detweiler, D. K., Hubben, K., and Patterson, D. F. (1960). Survey of cardiovascular disease in dogs-Preliminary report on the first 1,000 dogs screened. Amer. J. vet. Res., 21, 329.

Doyle, J. T., Patterson, J. L., Warren, J. Y., and Detweiler, D. K. (1960). Observations on the circulation of domestic cattle. Circulat. Res., $8,4$.

Glazier, D. B., and Kealy, J. K. (1960). Tetralogy of Fallot. Irish vet. J., 14, 108.

Hahn, A. W. (1908). Beitrag zur Anatomie der Kammerscheidewand unserer Haustiere. Dissertation, Bern (Vet. Med. Faculty). Jasper, D. E. (1948). Myocardial abscesses associated with a congenital cardiac defect in a heifer. Cornell Vet.,
38, 436. 
Meredith, J. H., and Clarkson, T. B. (1959). Tetralogy of Fallot in the dog. J. Amer. vet. med. Ass., $135,326$.

Roberts, S. J., Kennedy, P. C., and Delehanty, D. D. (1953). A persistent right aortic arch in a Guernsey bull. Cornell Vet., 43, 537.

Van Nie, C. J. (1961). Congenital Heart Malformation of the Pig, p. 45. Uitgeverij Ceres-Meppel.

Wensvoort, P. (1959). Der Tetralogie van Fallot, met atresia van der arteria pulmonalis, bij het hart van een Shetland pony. T. Diergeneesk., 84, 939.

Wood, P. (1956). Diseases of the Heart and Circulation, 2nd ed. Eyre and Spottiswoode, London. 